\title{
Structural factors in figure perception
}

\author{
JAMES L. McCLELLAND and JEFF MILLER \\ University of California, San Diego, La Jolla, California 92093
}

\begin{abstract}
In this report, we examine tachistoscopic perception of lines in structured figures. Previous research has shown that perception of lines is facilitated when the lines occur as parts of coherent three-dimensional figures. Experiment 1 demonstrates that it is not necessary for the figure to be three-dimensional to obtain facilitation. Experiments 2 and 3 show that three-dimensionality is not sufficient either. What is important is that the target lines be structurally relevant to the figure. However, structural relevance of a target segment to the figure as perceived with unlimited viewing time is not perfectly correlated with perceptibility under tachistoscopic conditions; it appears that the targets which fall on the external contour of a figure may be facilitated even without a high degree of structural relevance. In view of this, we suggest a model in which perceivers use processing heuristics to direct processing to aspects of the input that are potentially important for determining the structure of the final figure, working primarily from the outside in.
\end{abstract}

The perception of a visual target depends on the context in which the target is embedded (Banks \& Prinzmetal, 1976; Bell \& Handel, 1976; Biederman, 1972; McClelland, 1978; Pomerantz, Sager, \& Stoever, 1977; Pomerantz, 1977; Prinzmetal \& Banks, 1977; Weisstein \& Harris, 1974; Weisstein \& McGuire, 1978; Williams \& Weisstein, 1978; Womersley, 1977). For example, Weisstein and Harris (1974) have shown that detection of target lines is more accurate when they are embedded in wholistic, three-dimensional objects than when they are presented in less coherent, flatter patterns. Indeed, Williams and Weisstein (1978) have shown that under some conditions the detection of a single line segment can be more accurate when the segment is embedded in a figure than when the segment is presented alone. In their experiments, subjects were required to discriminate the four lines shown in Figure 1, either presented alone or presented in the context of a coherent three-dimensional figure. Several experiments found $11 \%$ to $15 \%$ greater accuracy when the lines were presented in the object context than when the lines were presented alone.

An even more dramatic effect of the same type has been obtained by McClelland (1978). His subjects viewed the Weisstein-Harris stimuli under conditions of pre- and posttarget masking using a line mask consisting of an array of unrelated lines similar to the mask shown in Figure 2. Under these viewing

This research was supported by NSF Grant BNS76-14830 to the first author and by Grant PHS NH 15828 to the Center for Human Information Processing, University of California, San Diego. We would like to thank Marguerite Moreno and Diana Wear for their assistance in subject running, data analysis, and figure preparation. Requests for reprints may be sent to either author at the Department of Psychology C-009, University of California, San Diego, La Jolla, California 92093. conditions, McClelland found a $34 \%$ advantage for lines in objects over single lines.

In this study, we will use the line masking conditions of McClelland as a tool for exploring the structural interactions that take place in perceiving wholistic figures. In particular, we will consider the structural factors responsible for the object-line effect (Williams \& Weisstein, 1978). First, we will ask what structural characteristics of a figure are important in facilitating the perception of components of the figure. Second, we will consider how the relation between the whole figure and the target component influences the facilitation.

\section{EXPERIMENT 1}

As a starting point, we will examine the contribution of three-dimensionality of a figure to the perception of lines presented within it. The importance of this factor has been considered in other recent studies. Womersley (1977) suggested that threedimensionality rather than coherence was important, since he found that a coherent three-dimensional figure was superior to an incoherent flat figure in one study, but found no difference between two flat figures differing in coherence in another. His results are not conclusive, however. In addition to the problem of acceptance of the null hypothesis, there may be a confounding factor at work. Womersley's coherent stimuli had more lines in the vicinity of the target lines than did the noncoherent stimuli. Therefore, it is possible that lateral interference (Eriksen \& Rohrbaugh, 1970; Estes, Allmeyer, \& Reder, 1976; Wolford, 1975) counteracted a possible coherence effect.

Williams and Weisstein (1978) also considered the importance of three-dimensionality for the object- 
Targets

1

Single Lines

Filled Crosses
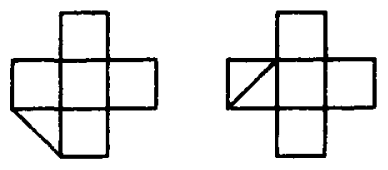

Unfilled Crosses
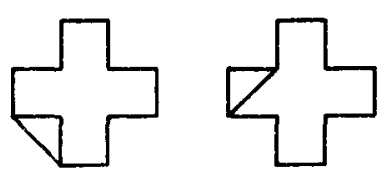

Ob jects

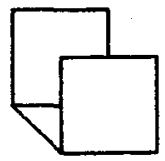

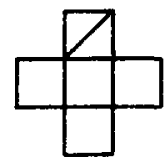
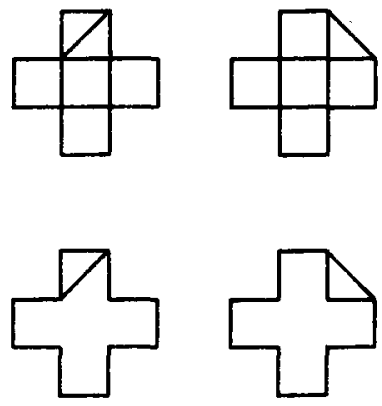

3

4
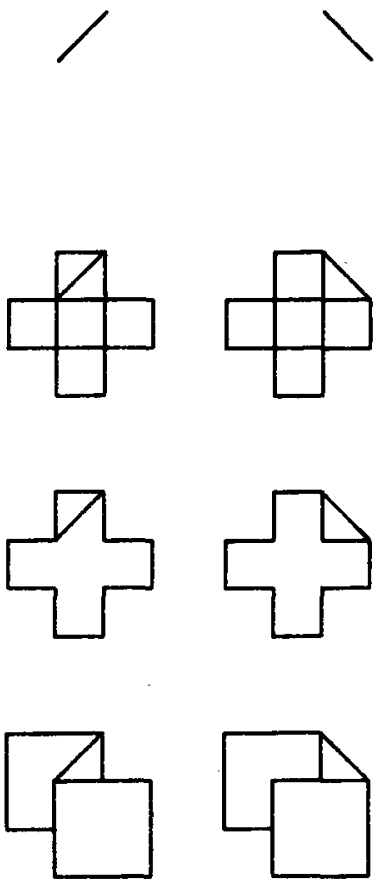

Figure 1. The target displays used in Experiment 1. The single line and object stimuli were originally used by Weisstein and Harris (1974).

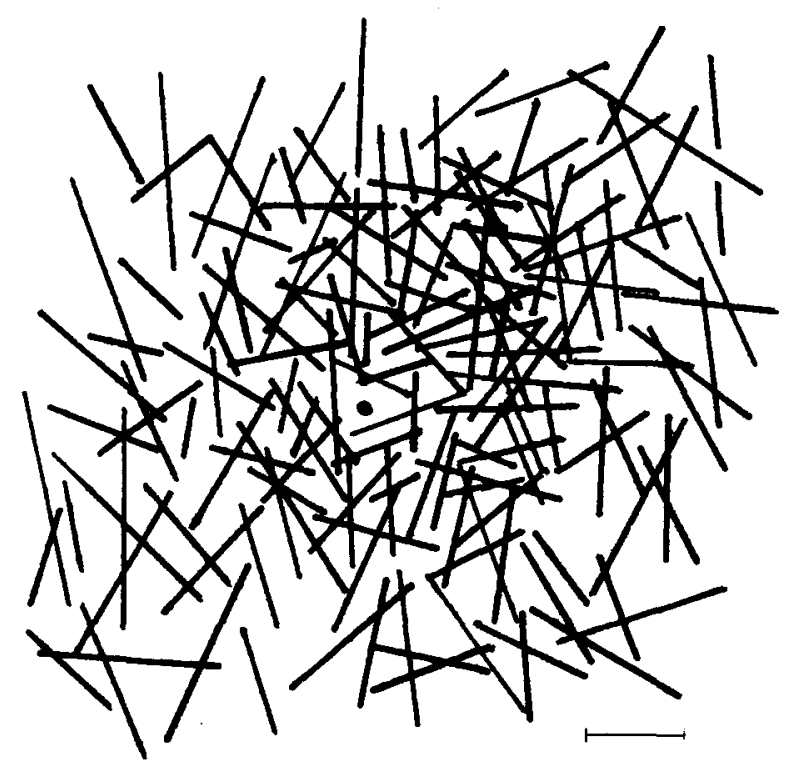

Figure 2. The masking stimulus used in Experiments 1 to 3. The line added at the lower right corresponds to $1 \mathrm{~cm}$ at the scale of the original figure. line effect. They constructed a control context intended to look less three-dimensional than the object context. Performance on this context was very nearly as good as performance in the object context, but they were unable to draw any firm conclusion because several subjects reported that this context looked somewhat three-dimensional under experimental viewing conditions.

The present experiment reexamines the role of threedimensionality by comparing perception of lines in perceptually flat "cross" contexts (Figure 1) to perception of single lines and lines in objects, using the line-mask conditions of McClelland (1978). Perceptual flatness of the cross contexts was checked by obtaining ratings from independent groups of subjects.

\section{Method}

Subjects. Eight naive UCSD undergraduates participated in the experiment in partial fulfillment of a course requirement. Each subject served for a single experimental session lasting slightly less than $2 \mathrm{~h}$.

Stimuli. As baselines for comparison, we used the single line and object conditions used by McClelland (1978) and Williams and Weisstein (1978). The two new contexts were two similar versions of a cross pattern which seemed to us to be both coherent and flat. Ideally, we wanted the cross contexts to be as similar 
as possible to the three-dimensional contexts, especially with respect to the lines touching or neighboring the target lines, in order to minimize differences in lateral interference. Since we were only able to approach this ideal, we used the two different cross contexts shown in the figure. These contexts differ primarily in local information surrounding the target segments, and provide a partial check on the magnitude of lateral interference effects. Ratings by independent subjects (see Appendix) confirmed our intuitions that these stimuli are indeed very low on the scale of three-dimensionality. Different groups rated the stimuli for wholistic three-dimensionality, local three-dimensionality in the vicinity of the target segment, and the structural relevance of the target segment to the figure considered as a whole (considered in Experiments 2 and 3). Three-dimensionality ratings for lines in the crosses pattern were much lower than the ratings for lines in objects and only very slightly higher than ratings for a set of bisected square stimuli included in the rating task for comparison purposes.

The target displays and the mask were composed of black lines on a white background. The displays were presented and their duration controlled by a four-field Gerbrands tachistoscope with standard lamps. The luminance of a blank card in both the target and mask fields was about $10 \mathrm{~cd} / \mathrm{m}^{2}$, as measured by a spectra Spotmeter. The line segments were about $.03^{\circ}$ wide at the viewing distance of $90 \mathrm{~cm}$, and the target segments were about $.7^{\circ}$ of visual angle in length. The line mask (shown in Figure 2) was composed of a haphazard pattern of lines of various lengths and orientations, following the procedure of McClelland (1978). The mask contained a fixation dot placed so as to coincide with the center of the complete square in the object context.

Procedure. Each subject was initially familiarized with the target lines and the appropriate responses, in each of the context conditions. The responses were just the digits "one" to "four" with "one" referring to the displays having the critical segment in the lower left, and with the other digits applying to the displays having the critical segment in successive locations proceeding in a clockwise direction around the fixation point. This was followed by nine blocks of 32 trials/block, of which the first three blocks were considered practice and were used to adjust the exposure duration to reach an average level of about $75 \%$ correct. Each block contained two presentations of each critical target segment in each context condition, with the order of presentation randomized separately for each block. Further adjustments in the exposure durations were made between blocks if performance deviated much from $75 \%$ correct averaged over all display types. The mean target duration averaging across subjects and blocks was $77 \mathrm{msec}$.

Each trial began with the subject fixating the round dot in the mask and initiating target onset with a buttonpress. Target duration was set by the experimenter, as described above. After target offset, the mask immediately reappeared, and the subject attempted to identify the target, guessing if necessary. The subjects were permitted to refer to a sheet which pictured all of the target displays (four different targets for each of four different context conditions) paired with the appropriate responses. This sheet was placed on the table in front of the subjects, and could be viewed by glancing down from the eyepiece of the tachistoscope. The subjects tended to make use of the sheet during familiarization, and some continued to use it during practice, but by the time the test phase of the experiment began very few of the subjects continued to rely on the sheet. The experimenter provided feedback after each trial by saying "good" if the subject was correct or by naming the correct response if the subject was incorrect.

\section{Results}

The attempt to find a flat context which can facilitate identification of the target lines relative to the single line condition was successful. However, the cross contexts used here produced weaker facilitation than the object context. Table 1 shows the average
Table 1

Experiment 1: Percentage Correct Identification for Each of the Four Target Lines in Each Context

\begin{tabular}{lcccc} 
& \multicolumn{4}{c}{ Context } \\
\cline { 2 - 4 } Target & & \multicolumn{2}{c}{ Cross } \\
\cline { 2 - 5 } Line & Line & Filled & Unfilled & Object \\
\hline 1 & .344 & .833 & .854 & .969 \\
2 & .635 & .594 & .594 & .927 \\
3 & .490 & .448 & .615 & .865 \\
4 & .573 & .813 & .906 & .927 \\
Average & .510 & .672 & .742 & .922 \\
\hline
\end{tabular}

percentages of correct responses for each of the four target lines in each of the four stimulus contexts. Both the main effect of context $[F(3,21)=30.4$, $\mathrm{p}<.01, \mathrm{MSe}=.03]$ and the main effect of target line $[\mathrm{F}(3,21)=6.3, \mathrm{p}<.01, \mathrm{MSe}=.038]$ were significant, as was their interaction $[F(9,63)=3.73$, $\mathrm{p}<.01, \mathrm{MSe}=.033$ ]. A comparison between the averages for the four contexts using Tukey's test (Keppel, 1973) showed that the cross contexts did not differ from each other $(p>.10)$, while both cross contexts were better than the single lines but worse than the objects $(p<.01)$.

Inspection of the target line by context interaction, shown in Table 1, reveals that a considerable portion of the significant interaction is due to special difficulty with Target Lines 2 and 3 in the cross contexts. These lines are the ones which appear in the interior of the figures, and they are identified no better in the cross contexts than when presented alone, though they are greatly facilitated by the object context. The slight performance difference between the two cross contexts is not consistent either over the two line segments in question or over subjects. Lines 1 and 4 , which appear on the outside of both the object and cross contexts, are identified much better in the cross contexts than alone. In short, the cross contexts facilitate identification only of the exterior target lines, while the object context facilitates all targets. Still, the exterior target lines are identified better in the object context (.948) than in the flat contexts $(.852)$, and this advantage is reliable across subjects $(t=3.24, p<.01)$.

Confusion matrices were examined in order to check on the possibility that the different contexts produced different patterns of errors. Errors on internal and external target lines were classified according to the relation between the target and the incorrect response, as shown in Table 2 . It is apparent that the distribution of errors does not vary dramatically across contexts.

\section{Discussion}

The superiority of performance with the cross contexts compared to the single lines demonstrates that flat contexts can indeed produce some facilitation. Since the ratings confirm the perceptual flatness of 
Table 2

Analysis of Errors (in Percentages), Experiment 1

\begin{tabular}{lcccc}
\hline & \multicolumn{4}{c}{ Context } \\
\cline { 3 - 4 } & & \multicolumn{2}{c}{ Cross } \\
\cline { 3 - 4 } & Line & F & U & Object \\
\hline Internal Target Confused With: & & & & \\
$\quad$ Other Internal Line & 61 & 45 & 52 & 50 \\
$\quad$ Adjacent External Line & 26 & 30 & 35 & 40 \\
$\quad$ Nonadjacent External Line & 13 & 25 & 13 & 10 \\
Number of Errors & $(84)$ & $(76)$ & $(92)$ & $(20)$ \\
External Target Confused With: & & & & \\
$\quad$ Other External Line & 26 & 39 & 21 & 30 \\
$\quad$ Adjacent Internal Line & 40 & 39 & 53 & 40 \\
$\quad$ Nonadjacent Internal Line & 34 & 22 & 26 & 30 \\
Number of Errors & $(104)$ & $(10)$ & $(34)$ & $(23)$ \\
\hline
\end{tabular}

Note $-F=$ filled,$U=$ unfilled .

these figures, it would appear that three-dimensionality of a figure is not a necessary condition for the object-line effect. While the data are consistent with a partial role for three-dimensionality, it appears that connected, coherent figures of either two or three dimensions may facilitate perception of their parts.

It would be possible to defend the view that threedimensionality is actually critical for facilitation by arguing that the cross contexts might appear threedimensional when presented under the viewing and instructional conditions of this experiment, as opposed to those prevailing under the conditions of the judgment task. It is worth noting, however, that the subjects were familiarized with the stimulus set prior to the beginning of tachistoscopic testing under normal viewing conditions. In addition, they chose their responses during the early phases of the experiment from a display viewed without masking and under normal room illumination. It seems likely, then, that the subjects went into the experiment thinking of the cross stimuli as flat (indeed, they were described that way by the experimenter).

There is a somewhat more plausible explanation of the present results in which three-dimensionality plays an important role. It could be that the facilitation by a context is due to early pickup of features that are consistent with a three-dimensional figure even though further processing would indicate that the figure is flat. This might be the case if, for example, subjects used processing heuristics which were designed to extract features revealing the (potentially three-dimensional) structure of the figure. We will return to this and related possiblities in the general discussion below.

How are we to interpret the advantage for objects over lines in the cross contexts? One possibility, of course, is that this difference is due to differences in the apparent three-dimensionality of the figures, or in their coherence as wholes. However, there is an alternative possibility having to do with a difference in the structural roles of the target lines. We will consider this possibility explicitly in the next experiment.

\section{EXPERIMENT 2}

In most studies of the effects of context on identification of targets, coherent contexts have been used with target lines that are structurally relevant to the context figure. That is, the target line plays a role in determining the object which the target represents, and is not merely an incidental addition to that object. In the object context, for example, the target lines determine whether the figure depicts an object with a floor on it, one with a ramp rising through it, one with a diagonal divider across it, or one with a side wall on the right. It is possible that the perceptibility of a line segment depends on the degree to which it contributes to the perceived structure of the figure. This hypothesis was in fact suggested to us by the results of Experiment 1. The target lines in the cross contexts did not appear to be particularly structurally relevant, and the ratings of structural relevance support this view. Perhaps at least part of the reason for the difference in perceptibility of these lines compared to the objects was that they simply failed to contribute as much to a determination of the form of the whole in which they were imbedded. Experiment 2 was designed to test more directly for an effect of structural relevance, holding the context the same and varying the target segments.

\section{Method}

Subjects. The subjects were 32 undergraduates naive to the purposes of the experiment, and they received course credit for their participation.

Stimuli. Two different contexts were used: the object context and a new context called the corner-square context. The latter was originally intended to serve as a flat context closely matched to the object context for contours in the vicinity of the target segment. However, subjects rated these figures significantly higher in local three-dimensionality than the simple square included as a baseline for flatness, so we must treat the corner-square stimuli as somewhat three-dimensional in appearance.

For both the object and the corner-square context, two different types of target lines were used (Figure 3). One type, Target Lines 2 and 3 ,from the preceding experiment, was considered to be highly structurally relevant to the target, while the other type was a pair of lines which seemed to us to be completely incidental to the figure considered as a whole. Indeed, the structural relevance ratings given to these items by our rating subjects were quite low in comparison to the ratings given to the relevant target items, especially in the case of the object context.

Design. Each subject was randomly assigned to one of four conditions, varying according to the instructions given to the subject (wholistic vs. target) and whether or not the target lines were structurally relevant to the contexts. Subjects in the wholistic instructional conditions were instructed to try to see the stimuli as whole three-dimensional forms, while subjects in the target instructional condition were simply told to focus on identifying 


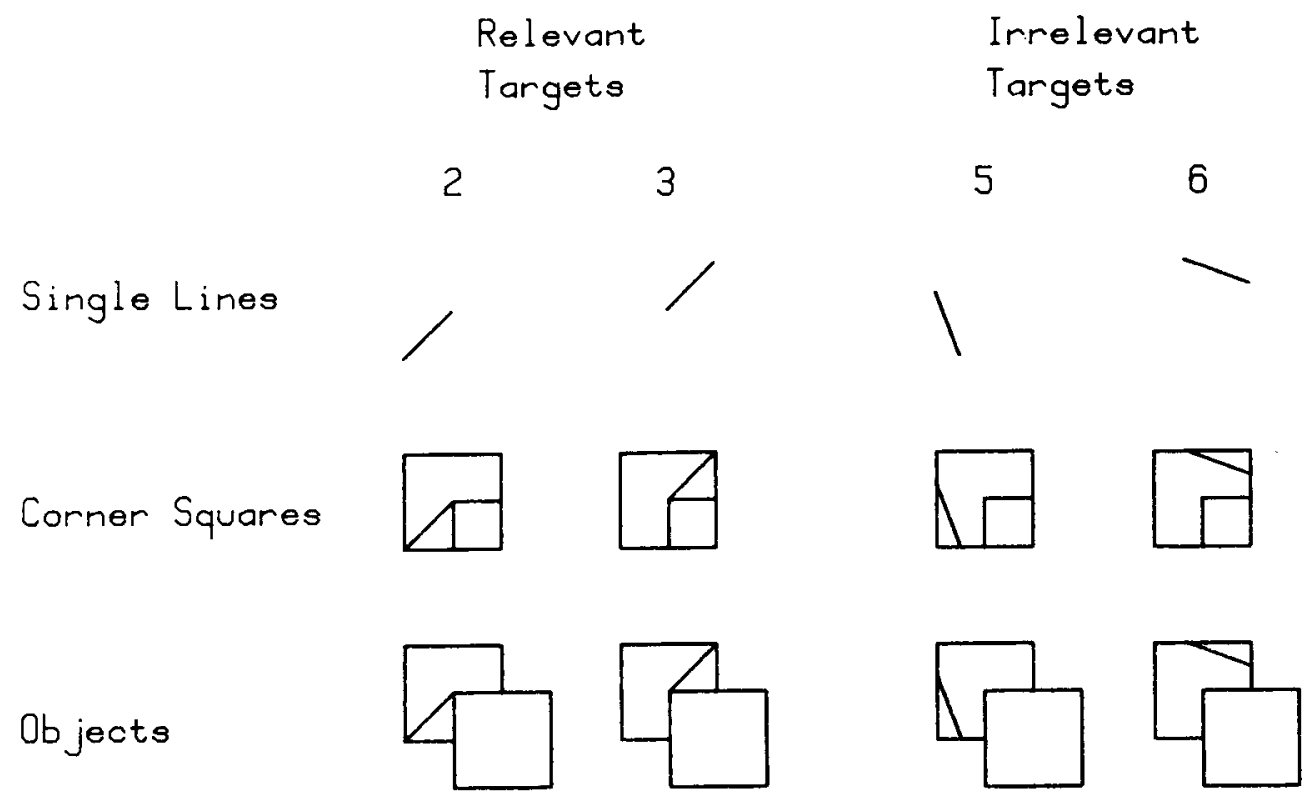

Figure 3. The target displays used in Experiment 2.

which of the two targets was presented, regardless of context. The intent of the instructional manipulation was to determine whether the set to attend to the whole figure or just to the target segment would influence the magnitude of any contextual facilitation effects (cf. Johnston \& McClelland, 1974). As this manipulation produced no significant effects or interactions, it will not be considered in the presentation of the results.

Subjects in the structurally relevant conditions saw only the stimuli in which the target lines were structurally relevant to the contexts, while subjects in the other groups saw only the stimuli in which the targets were structurally irrelevant.

Procedure. Details of the procedure were nearly identical to those of Experiment 1, with the exception of the insertion of the instructional manipulation already described. In this experiment, the subjects were given 96 practice trials before the beginning of the experiment, and there were eight experimental blocks of 24 trials/block. The mean target duration was $68 \mathrm{msec}$.

\section{Results and Discussion}

The two different types of target segments produced very different results (Table 3 ). An analysis of variance on the percent correct data revealed no significant main effects, but the interaction of structural relevance of the target line and type of context was significant $[\mathrm{F}(2,56)=9.3, \mathrm{p}<.01, \mathrm{MSe}=$ $.015]$. This interaction results from an advantage for both objects and squares over single lines in the structurally relevant condition with a reversal of these effects in the irrelevant condition $(p<.05)$.

\section{EXPERIMENT 3}

While the results of Experiment 2 support the conclusion that structural relevance is an important determiner of facilitation, it is possible to construct other explanations of the results based on the idea that the particular target segments used in the irrelevant target condition are insensitive to perceptual facilitation. To rule out any such possibilities, we generated a new context (Figure 4) called the hurdles context in which these segments appear to be highly relevant to the target display. These stimuli were developed after the structural relevance rating experiment was run, so we do not have ratings on them, but the target segments determine whether the figure is an upright hurdle or a mirror image of the same hurdle fallen over on its side, so it is apparent that the target segments are structurally relevant in this context. Clearly, if this context produces facilitation, we can set aside the insensitivity hypothesis.

A second goal of Experiment 3 was to show that structural relevance is important, even when the target segment occurs in a highly three-dimensional context. Experiment 2 failed to make this point clearly because the object context appeared less three-dimensional when it contained an irrelevant

Table 3

Experiment 2: Percentage Correct Identification as a Function of Structural Relevance and Type of Context

\begin{tabular}{ccccc}
\hline & \multicolumn{3}{c}{ Context } \\
\cline { 2 - 4 } & \multicolumn{2}{c}{ Square } & \\
\cline { 2 - 5 } Target Type & Line & Corner & Object & Duration \\
\hline Relevant & 64.7 & 75.2 & 82.1 & 65 \\
Irrelevant & 80.6 & 71.1 & 73.2 & 72 \\
\hline
\end{tabular}

Note-Direct comparison of performance levels across relevance conditions is misleading since relevance was a between-subjects variable and exposure durations were adjusted for each subject to obtain approximately $75 \%$ correct performance averaging over context conditions. 


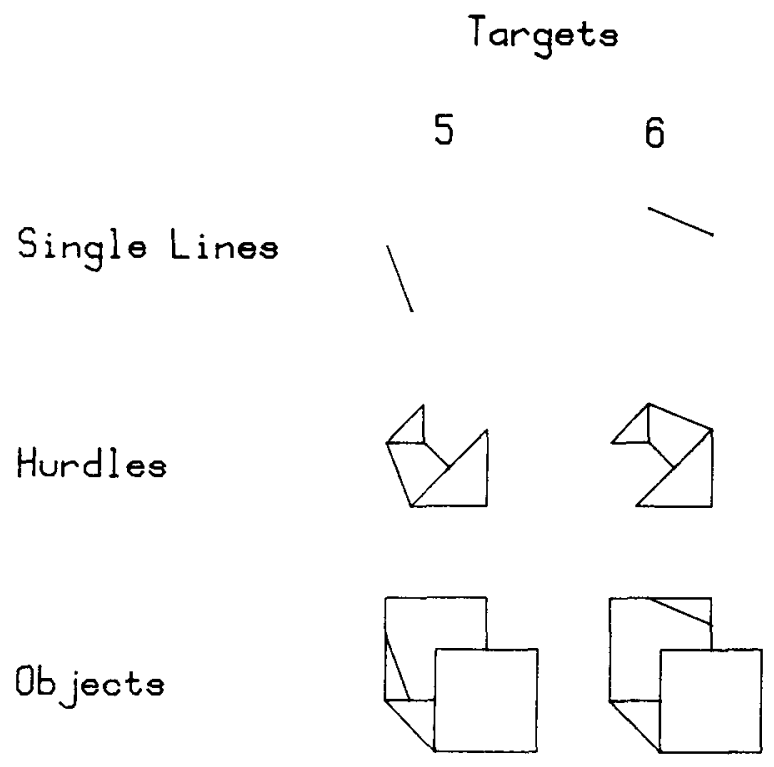

Figure 4. The target displays used in Experiment 3.

target in the place of one of the relevant ones. To get around this difficulty, we used a new version of the object context, which itself includes one of the structurally relevant target segments. These stimuli were included in the ratings, and subjects rated them just as three-dimensional as the object targets without the irrelevant lines added. If three-dimensionality of the overall figure formed by the context and the target is sufficient to facilitate identification of the target, then there should be an advantage in the object condition in this experiment. On the other hand, if structural relevance is a determining factor, the target lines should produce facilitation for the hurdles context, but not for the object context.

\section{Method}

Subjects. Eight naive undergraduates were recruited to serve as subjects in this experiment, and they received course credit or $\$ 2.50$ for their participation.

Procedure. Details of the procedure in this experiment were identical to those of Experiment 2, except that a single group of subjects was run and all subjects were instructed to try to perceive the target segments as accurately as possible. As in Experiment 1 , there was no specific instruction to either focus in on the target or to try to perceive it as a whole. The mean target duration was $70 \mathrm{msec}$.

\section{Results and Discussion}

The hurdles context facilitated perception of the target lines compared to the single-line control condition, but the complete object context did not. Forcedchoice responses were $94 \%$ correct for the hurdles, $60 \%$ for the single lines, and $62 \%$ for the lines in objects. Analysis of variance revealed that the main effect of target type was highly significant $[F(1,7)$ $=43.99, \mathrm{p}<.001, \mathrm{MSe}=.006 \mathrm{]}$, and Dunnet's test for comparisons of multiple experimental groups against a control revealed that the difference between the hurdles and the single lines condition was significant $(p<.01)$, while the difference between the objects and the single lines was not. We can conclude, then, that three-dimensionality of the target figure in which a line occurs is insufficient for facilitation. Instead, the experiment underscores the importance of structural relevance.

\section{GENERAL DISCUSSION}

Our experiments have replicated the large object advantage over single lines obtained previously by McClelland (1978) under similar masking conditions and by Williams and Weisstein (1978) with no mask. The results also extend these findings by showing that the effect can be obtained with other types of contexts. Exterior target lines in a flat cross pattern were perceived more accurately than single lines, although internal lines in this context did not show any advantage. We also found that internal lines in the rather flat corner square context had some advantage over single lines. Finally, we obtained facilitation for a new set of target lines in a context which looks like a hurdle in an upright or fallen position depending on which target segment is present.

Taken as a whole, these results provide little reason to continue to hold the view that three-dimensionality of the figure within which the target is embedded, or local three-dimensionality in the area of the target segment itself, is necessary to obtain perceptual facilitation. The ratings of the cross pattern for wholistic three-dimensionality were only slightly higher than our simple square control pattern, and the ratings of these stimuli for local three-dimensionality were even lower and closer to the ratings of the control. Thus, the advantage for external line segments in the cross contexts is difficult to attribute to the effect of the context in producing an apparently three-dimensional structure. Even more certain is the conclusion that three-dimensionality of the whole is not sufficient for facilitation. This is clear from the finding of Experiment 3 that structurally irrelevant targets in the highly three-dimensional object context produced no facilitation, even though the same segments produced a very strong facilitation effect in another context.

On the positive side, we have evidence that facilitation is dependent on the structural relevance of a target to the figure in which it is presented. Irrelevant internal lines in our object and square contexts did not have an advantage over the same line segments in isolation, even (as just mentioned) when the figure was highly three-dimensional, while relevant internal and external lines in these same contexts were facilitated. We can rule out the possibility that the difference between relevant and irrelevant internal lines was simply due to some idiosyncratic insensitivity to facilitation for the particular irrelevant internal lines used, since these same lines produced 
a strong facilitation effect when they were placed in the hurdle context.

The relation between structural relevance and degree of facilitation is not quite perfect, however. The external lines in the cross context had a large advantage over single lines, while internal lines in the same context did not, even though both external and internal targets in the cross context scored low in structural relevance.

One way to make sense out of the pattern of results is to view the perceptual process as an ongoing series of operations in which the results of early analyses may be used to direct later analyses. Assuming that structural relevance judgments reflect the end product of perceptual performance, it is apparent that these judgments may be only loosely related to the operation of the early perceptual processes that determine what is seen in a tachistoscopic presentation. What the viewer sees in such a case may depend, at least in part, on the application of processing heuristics designed to aid in the rapid construction of structural descriptions of wholistic stimuli (Waltz, 1975). Waltz's heuristics suggest possible reasons why performance should be particularly poor on irrelevant, internal target lines, as opposed to equally irrelevant target segments lying on the external contour of the figure.

The most basic heuristic suggested by Waltz is to work from the outside of the figure inward, establishing first its external contour and only then analyzing the figure for internal details. This heuristic would account for the advantage of external over internal lines in the cross contexts, and may account in part for the very large facilitation produced by the hurdles context, in which the targets fell on the external contour of the figure. There is also some indirect indication that this "outside-in" strategy is operating with the objects as well: In Experiment 1, the object advantage over single lines was $49 \%$ for external lines (1-4) but only $33 \%$ for internal lines (2-3). Further, in a preliminary experiment using a slight variant of the mask used in Experiment 1, a $15 \%$ advantage for external lines over internal lines in objects was found as well. The small size of the direct advantage for external over internal lines in Experiment 1 may be due to a combination of a ceiling effect operating on the objects, and some local mask property making internal lines relatively easy in isolation.

The outside-in heuristic must be supplemented, of course, to account more fully for our results. Another useful heuristic suggested by Waltz is one in which processing is directed to lines intersecting with corners of the external contour before lines which intersect with the sides of the external contour. If we add this heuristic, we would be able to account for the effects of structural relevance in Experiments 2 and 3. However, this heuristic is not enough to account for all of our results. The finding that internal lines in objects are perceived more accurately than the same lines in the corner square context or the cross context would suggest that the process of construction is guided by more subtle properties of the stimuli than we have been able to identify thus far.

Even the simple heuristics we have suggested depend for their function on specification of the relevant contours; the ability to direct processing to contours which intersect the corners of a figure presupposes that the locations of these corners have been determined. In view of this, perhaps the most plausible framework for a model is one in which the constructive process is guided by what has already been extracted. Such a system could be based on progressively more refined heuristics designed to facilitate the construction of structural descriptions of the types of objects we normally encounter. Perception may begin with a global process which segregates the figure as a whole from the background (Neisser, 1967), perhaps on the basis of an analysis of the low spatial frequency information in the display (Breitmeier and Ganz, 1976; Navon, 1977). The figure thus segregated could then be analyzed, with most processing capacity initially devoted to the outside contour of the figure. As the vertices on the external contour of the figure became established, further processing could be directed toward those areas of the figure which appeared most promising for further specification of the structure of the figure.

How can we explain in the context of this sort of model the difficulty subjects have in perceiving single lines? One possibility is that these stimuli simply fail to engage the constructive mechanism as effectively as larger connected figures. One reason for this (although perhaps not the only one) may be their lack of low spatial frequency components. A mechanism which relied on low spatial frequency information to isolate the figure to be processed might account for the object advantage over single lines under no-mask conditions (Williams \& Weisstein, 1978) as well as under the masking conditions of the studies reported here.

It is beyond the scope of the present paper to present a full discussion of a model of this constructive process. However, the suggestions we have borrowed from Waltz's (1975) computer scheme for analyzing line drawings of scenes may provide a starting point for further experimental investigations examining how the construction of a representation of a line drawing actually takes place in the human observer.

\section{REFERENCES}

Banks, W. P., \& Prinzmetal, W. Configurational effects in visual information processing. Perception \& Psychophysics, 1976, 19, 361-367.

BeLl, H. H., \& Handel, S. The role of pattern goodness in the reproduction of backward masked patterns. Journal of Experimental Psychology: Human Perception and Performance, 1976, 2, 139-150. 
Biederman, I. Perceiving real world scenes. Science, 1972, 177, 77-80.

BrittMEYER, B. G., \& GANZ, L. Implications of sustained and transient channels for theories of visual pattern masking, saccadic suppression, and information processing. Psychological Review, i 976, 83, 1-36.

Eriksen, C. W., \& Rohrbaugh, J. Visual masking in multielement displays. Journal of Experimental Psychology, 1970, 83, 147-154.

estes, W. K., Allmeyer, D. H., \& Reder, S. M. Serial position functions of letter identification at brief and extended exposure durations. Perception \& Psychophysics, 1976, 19, 1-15.

Johnston, J. C., \& McClelland, J. L. Perception of letters in words: Seek not and ye shall find. Science, 1974, 184, 1192-1194.

KE PPEL, G. Design and analysis: $A$ researcher's handbook. Englewood Cliffs, N.J: Prentice-Hall, 1973.

MCClelland, J. L. Perception and masking of wholes and parts. Journal of Experimental Psychology: Human Perception and Performance, 1978, 4, 210-223.

Navon, D. Forest before trees: The precedence of global features in visual perception. Cognitive Psychology, 1977, 9, 353-383.

NeISSER, U. Cognitive psychology. New York: AppletonCentury-Crofts, 1967.

Pomerantz, J. Pattern goodness and speed of encoding. Memory \& Cognition, 1977, 5, 235-241.

Pomerantz, J., Sager, L., \& Stoever, R. Perception of wholes and their component parts: Some configurational superiority effects. Journal of Experimental Psychology: Human Perception and Performance, 1977, 3, 422-435.

Prinzmetal, W., \& Banks, W. P. Good continuation affects visual detection. Perception \& Psychophysics, 1977, 21, 389-395.

WALTZ, D. Understanding line drawings of scenes with shadows. In P. Winston (Ed.), The psychology of computer vision. New York: McGraw-Hill, 1975.

Weisstein, N., \& Harris, C. S. Visual detection of line segments: An object superiority effect. Science, 1974, 186, 752-755.

Weisstein, N., \& McGuire, W. Computing the next step: Psychophysical measures of representation and interpretation. In E. Riseman \& A. Hanson (Eds.), Computer vision systems, New York: Academic Press, 1978.

Williams, A., \& Weisstein, N. Line segments are perceived better in a coherent context than alone: An object-line effect. Memory \& Cognition, 1978, 6, 85-90.

Wolford, G. Perturbation model for letter identification. Psychological Review, 1975, 82, 184-199.

Womfrsiey, M. A contextual effect in feature detection with application of signal detection methodology. Perception \& Psichophysics, 1977, 21, 88-92.

(Received for publication November 20, 1978; accepted for publication July 5, 1979.)

\section{APPENDIX}

Ratings of the Figures Used in Experiments 1-3

Three types of ratings were collected: ratings of the threedimensionality of the figures as whole units, ratings of local three-dimensionality, and ratings of the structural relevance of the target lines to the figures. Different groups of subjects were used for each type of rating.

\section{Method}

Subjects. The subjects were 180 undergraduates at UCSD, mostly students enrolled in an introductory psychology class. All were naive with respect to the purpose of the ratings.

Stimuli. The subjects were asked to rate all of the stimuli used in the experiments, with the exception of the hurdles used in Experiment 3 (these figures had not been invented at the time the ratings were obtained). Each subject rated the four objects, the eight stimuli formed from the four target lines and the two cross contexts used in Experiment 1, the four stimuli formed from the two square contexts and two target lines of Experiment 2, and the six stimuli with structurally irrelevant lines from Experiments 2 and 3. Also included for comparison purposes were four figures formed by putting horizontal, vertical, rising diagonal, or falling diagonal bisectors inside of a square.

Procedure. The subjects were generally run in groups of 8 to 15 , at the beginning of meetings of sections in their introductory psychology class. Each subject was given a booklet which had a single figure on each page and an instruction sheet describing the task. Sixty subjects were run in each of the three rating conditions. Subjects in the "wholistic three-dimensionality" group were asked to assign each figure a number between 0 and 99 "according to how three-dimensional it looks." For example and comparison purposes, these subjects were shown a square and told to assign it a rating of 20 , and were shown a perspective drawing of a cube which they were told to give a rating of 80 . Subjects in the "local three-dimensionality" group were asked to assign each figure a number between 0 and 99 " according to how threedimensional it looks in the vicinity of the particular line we specify." For this group, each page of the booklet had a word or phrase indicating the line that subjects were to look at in considering local three-dimensionality. The example figures shown to this group were a square and a cube, as for the wholistic group, and the subjects were told to give a rating of 20 to the left side of the square and a rating of 80 to the top diagonal line in the cube. Subjects in the "structural relevance" group were told to assign each figure a number between 0 and 99 "according to how structurally relevant the indicated line is to the figure." The first example figure for this group was a random jumble of lines of which subjects were asked to consider a particular one. They were told to give this line a low structural relevance rating of 20 , "since this line is fairly incidental to the figure, and the figure

Table 4

\begin{tabular}{|c|c|c|c|}
\hline \multirow[b]{2}{*}{ Context } & \multicolumn{2}{|c|}{ Three-Dimensionality } & \multirow{2}{*}{$\begin{array}{l}\text { Structura } \\
\text { Relevance }\end{array}$} \\
\hline & Holistic & Local & \\
\hline Object (1-2) & 75.0 & 78.5 & 75.7 \\
\hline Unfilled Cross (1) & 31.4 & 22.9 & 34.4 \\
\hline Filled Cross (1) & 34.1 & 25.0 & 27.4 \\
\hline Corner Square with Relevant Target (2) & 33.7 & 34.8 & 44.0 \\
\hline Corner Square with Irrelevant Target (2) & 31.5 & 24.3 & 32.1 \\
\hline Object with Irrelevant Target (2) & 54.1 & 28.1 & 27.7 \\
\hline Complete Object with Irrelevant Target (3) & 70.0 & 30.6 & 36.2 \\
\hline Square with Bisector* & 24.5 & 20.2 & 67.2 \\
\hline None $(1-3)$ & 9.4 & 10.9 & \\
\hline
\end{tabular}

Note-Numbers in parentheses indicate experiments in which the context was used.

*This context served as a baseline context for comparison purposes (see text). 
does not seem to change in any essential way if the line is removed." The second example figure was a square, and subjects were told to give a high structural relevance rating of 80 to the left side, since this line "is not incidental and the figure would look very different without the line."

\section{Results}

Table 4 shows the average rating for each of the contexts for each type of rating. These averages are computed across subjects and across target segments within a context. Preliminary one-way analyses of variance of the ratings of individual targets within contexts was performed on each of the contexts separately, for each type of rating. Of the 26 significance tests thus performed, only four were signifi- cant $(.01<\mathrm{p}<.05)$, the largest difference was only 8 points on the 100-point scale, and there was no apparent pattern to the differences.

For each set of ratings, a one-way analysis of variance was performed on the average values for the $\mathbf{1 0}$ different contexts in Table 4. In each case, the contexts differed reliably $(\mathrm{p}<.001)$. Tukey $5 \%$ critical range values were computed in order to make comparisons among the pairs of contexts, and the resulting values were 7.1, 9.8, and 8.1 for the wholistic three-dimensionality ratings, local threedimensionality ratings, and structural relevance ratings, respectively. The comparisons of interest are discussed in the main body of the text where relevant. 